\title{
Aged rats under zoledronic acid therapy and oral surgery
}

\author{
Mariza Akemi Matsumoto ${ }^{\text {a, * }}$, Elisa Mara de Abreu Furquim ${ }^{\text {a }}$, Alaíde Gonçalves ${ }^{\text {, }}$ \\ Joel Ferreira Santiago-Júnior ${ }^{\text {b }}$, Patrícia Pinto Saraiva ${ }^{\text {b }}$, Camila Lopes Cardoso ${ }^{\text {b }}$, \\ Marcelo Salles Munerato ${ }^{\mathrm{b}}$, Roberta Okamoto ${ }^{\mathrm{a}}$ \\ a São Paulo State University (Unesp) (Head: Prof. W. R. Poi), School of Dentistry, Araçatuba, Rua José Bonifácio 1193, Araçatuba, SP, Brazil \\ ${ }^{\mathrm{b}}$ School of Dentistry, Sagrado Coração University - USC, Rua Irmã Arminda 10-50, Bauru, SP, Brazil
}

\section{A R T I C L E I N F O}

\section{Article history:}

Paper received 28 September 2016

Accepted 3 February 2017

Available online 17 February 2017

\section{Keywords:}

Aging

Bisphosphonate

Oral surgery

Rats

\begin{abstract}
A B S T R A C T
Introduction: Aging brings a number of health conditions that can compromise the healing process in elderly individuals, significantly when it comes to bone tissue. The aim of the present study was to analyze the effects of zoledronic acid (ZL) therapy on socket healing of aged male rats.

Materials and methods: Twenty-four Wistar male rats, 20 months old, underwent surgical procedures for the extraction of the upper right incisor and were divided into two groups according to the treatment: Control (C) - intravenous (IV) $0.9 \%$ saline, ZL $-0.035 \mathrm{mg} / \mathrm{kg}$ of IV zoledronic acid, both every 15 days. At the fifth dose of both substances, tooth extractions were performed and the animals were euthanized after 14 and 28 days.

Results: IV administration of ZL caused OPG-RANKL system imbalance, resulting in deficient bone formation and remodeling and alteration of osteoclast morphology, as well as maintaining persistent inflammation during the healing period.

Conclusions: IV administration of ZL delayed extracted dental socket healing of aged rats, but not enough to cause osteonecrosis, raising a question about different responses to IV BP therapy considering animal age.
\end{abstract}

() 2017 European Association for Cranio-Maxillo-Facial Surgery. Published by Elsevier Ltd. All rights reserved.

\section{Introduction}

An increasing elderly population has brought to the scene a number of age-associated health conditions, and among them are those involving the musculoskeletal system (Adam et al., 2003). On a physiological basis, senescence leads to bone cell disturbance, and the rate of bone formation decreases due to a reduction in proliferation and differentiation of multipotent stem cells, decrease of osteoblast life-span and osteocyte death (Manolagas and Parfitt, 2010; Marie, 2014). In addition, organic components also undergo deleterious alterations, and enzymatic crosslinks of type I collagen fibrils are gradually replaced by non-enzymatic glycosylation or oxidation induced by advanced glycation end products (AGEs), which disturb matrix mineralization and impair biological and mechanical properties of bone tissue (Allen et al., 2008).

\footnotetext{
* Corresponding author. Rua José Bonifácio 1193, CEP 16015-050, Araçatuba, SP, Brazil. Fax: +55 1836363200 .

E-mail address: vicmak.blv@terra.com.br (M.A. Matsumoto).
}

Along with the abovementioned unfavorable conditions, the risk of osteoporosis and malignant disease increases (Chan et al., 2016; Ostan et al., 2016), requiring specific drug therapy in order to protect bone tissue from uncontrolled resorptive processes. For this, particular preference to nitrogen-containing bisphosphonates (n-BPs) is observed in comparison to new drugs, especially considering their effectiveness and cost (Pazianas and Abrahamsen, 2016). Among this class of drug, zoledronic acid is the one of choice for metastatic breast and prostate cancer (Turner et al., 2016). An important related effect of its long-term use is the development of osteonecrosis of the jaw (ONJ), mainly triggered by invasive oral procedures or even with unfavorable oral conditions, such as periodontal diseases odontogenic infections, and improperly adapted prostheses (Marx, 2003; Ruggiero et al., 2009; Pelaz et al., 2015).

Despite the current information available about senescence and its consequent changes and demanding necessities, little is known about the dynamics of bone healing under this condition. In order to contribute to the understanding of the healing process, animal models play an important role, revealing differences in osteogenic 
gene expression and in biological potential (Alleyene et al., 2016; Ishijima et al., 2016).

The present study aimed to analyze bone repair of rats under a possible combination of conditions such as age, intravenous zoledronic acid therapy and oral surgery.

\section{Materials and methods}

All experimental protocols involving animals performed in this study were approved by the Ethical Committee for Animal Care of the Araçatuba School of Dentsitry - FOA/UNESP (protocol 2015/ 00209) and were performed in accordance with the European Communities Council Directive of 24 November 1986 (86/609/EEC), as well as of the Brazilian Society of Laboratory Animal Science (COBEA).

\subsection{Study design}

Twenty-four albinus Wistar male rats, mean age of 20 months and weight of $700 \mathrm{~g}$, underwent surgical procedures for the extraction of the upper right incisors, and were divided into two groups according to the treatment: Control group (C) - IV administration of $0.9 \%$ saline solution, ZL group (ZL) - IV administration of zoledronic acid (Novartis Pharma Stein AG, Stein, Swiss). Animals of the $C$ group received IV injections of $0.9 \%$ saline solution, whereas those of the ZL group were treated with $0.035 \mathrm{mg} / \mathrm{kg}$ IV zoledronic acid every 15 days (Hokugo et al., 2010) in the tail vein, until the end of the experiment. After the fourth dose of both substances, tooth extractions were performed. After 14 and 28 days from dental extractions, all animals were euthanized for specimen removal.

\subsection{Surgical procedure}

At the beginning of the surgical procedures the animals were sedated with intraperitoneal administration of $1 \%$ ketamine $(0.20 \mathrm{ml} / \mathrm{kg})$ and $2 \%$ xylazine $(0.30 \mathrm{ml} / \mathrm{kg})$ (Francotar, Virbac Ltda., São Paulo, Brazil). The animals were positioned in supine position to facilitate oral cavity access. Antisepsis of the oral mucosa was performed with topical $1 \%$ polyvinylpyrrolidone aqueous solution before tooth extraction. Animals first had their gingiva detached using specific retractors, and the teeth were dislocated from the alveolus with an adapted dental forceps. Then the sockets were washed with sterile $0.9 \%$ saline solution, and the blood clot formation was observed. No suture was performed. All animals were medicated with subcutaneous injection of $0.075 \mathrm{mg} / \mathrm{kg}$ of tramadol every $24 \mathrm{~h}$ for 3 days. After 14 and 28 days, all animals ( $\mathrm{n}=6$, per group and periods) were euthanized and the maxillae were removed and immediately immersed in $10 \%$ buffered formalin.

\subsection{Histological procedures and morphometric analysis}

The specimens were fixed up to $48 \mathrm{~h}$ before being washed in tap water for $24 \mathrm{~h}$. After, they were immersed in $4 \%$ EDTA solution for a period of 20-30 days for demineralization. When demineralization was achieved, the maxillae were divided and only the right side, containing the alveolus, underwent histotechnical procedures to be stained with hematoxylin and eosin (HE) and Goldner trichrome (GT). For microscopic analysis, inflammatory infiltrate, bone formation, maturing and remodeling, bone viability and the presence of microbial biofilm were observed. For quantitative analysis, 10 fields of each third part of the alveolus (cervical, medium, and apical) were registered, a total of 30 fields in $40 \times$ magnification, for the quantification of mononuclear and polymorphonuclear leukocytes of each field.

\subsection{Immunohistochemistry}

For the analysis of the proteins related to bone formation, maturing and remodeling, $3 \mu \mathrm{m}$ sections of each specimen were deparafinized in xylene and rehydrated in a graded series of ethanol to distilled water and immersed in $0.01 \mathrm{M}$ citrate-buffer at pH 6.0 to be heated in a steamer for $30 \mathrm{~min}$. Histological slices were treated with proteinase $\mathrm{K}$ for $30 \mathrm{~min}$ at room temperature. Endogenous peroxidase was blocked with $2 \%$ peroxide hydrogen for $10 \mathrm{~min}$ and washed with PBS (phosphate buffer solution). After this period, primary polyclonal antibodies (Santa Cruz Biotechnology, Santa Cruz, CA, EUA) anti-Runx2 and anti-RANKL, anti-OPG, anti-TRAP and anti-OC were incubated overnight at $4^{\circ} \mathrm{C}$ and washed for $30 \mathrm{~min}$, three times. The slices were incubated with biotinylated secondary antibody for $30 \mathrm{~min}$, washed in PBS and incubated with streptavidin-peroxidase conjugate (LSAB, Dakocytomation, Carpinteria, CA, USA) for $30 \mathrm{~min}$. Then, the reaction was stained with 3,3'-diaminobenzidine tetra hydrochloride (Sigma Aldrich, St Louis, MO, USA) and counterstained with Harris hematoxylin. For negative control, primary antibody was omitted. Labeling levels for each antibody were determined by semiquantitative analysis, considering the scores from "-" to " +++ " $(-=$ absent, $+=$ mild, $++=$ moderate, and $+++=$ intense) performed by two evaluations in a double-blind system (Luvizuto et al., 2010).

\subsection{Statistical assessment}

Data obtained from histomorphometric assessment were organized in a Table (Excel, Microsoft Office Excel, Redmond, WA, USA), and analyzed in relation to their normal distribution using ShapiroWilkes and Equal Variance tests, followed by 2-way ANOVA (Group and Period) and by Tukey test, in order to detect differences between the groups (SigmaPlot 12.0, San Jose, CA, USA). Significance level of $5 \%$ was adopted for the analysis.

\section{Results}

\subsection{Gross examination}

Extracted sockets of the $C$ group healed uneventfully, and at day 28 , they were totally covered by the mucosa. On the other hand, 2 out of 7 animals of the ZL group still presented an open wound at day 28; however, no bone exposure was observed (Fig. 1).

\subsection{Histological analysis}

Control Group - At day 14, the dental socket was filled by primary bone trabecullae presenting innumerous osteocytes, lined by active osteoblasts and by bone lining cells. In the medium region of the alveolus, mature bone trabecullae could be seen, presenting a mixture of irregular matrix surrounded by organized matrix, associated with osteoclasts. Among the trabecullae there was highly cellular loose connective tissue, infiltrated by mononuclear leukocytes in a diffuse distribution. Focal blood clots were also detected in medium and apical regions. At day 28, mature and remodeling bone was noted, exhibiting lamellar constitution and presenting bone lining cells on its surface. Reversal lines and bone multinucleated units (BMUs) were observed. Mononuclear leukocytes were also seen in the fibrous connective tissue and medullar tissue (Fig. 2).

ZL Group - After 14 days, bone trabecullae under a maturating process were observed, showing evident osteoclastic activity. Different from the $C$ group, bone maturing was diffuse, and not restricted to the central region of the sockets. In the cervical 

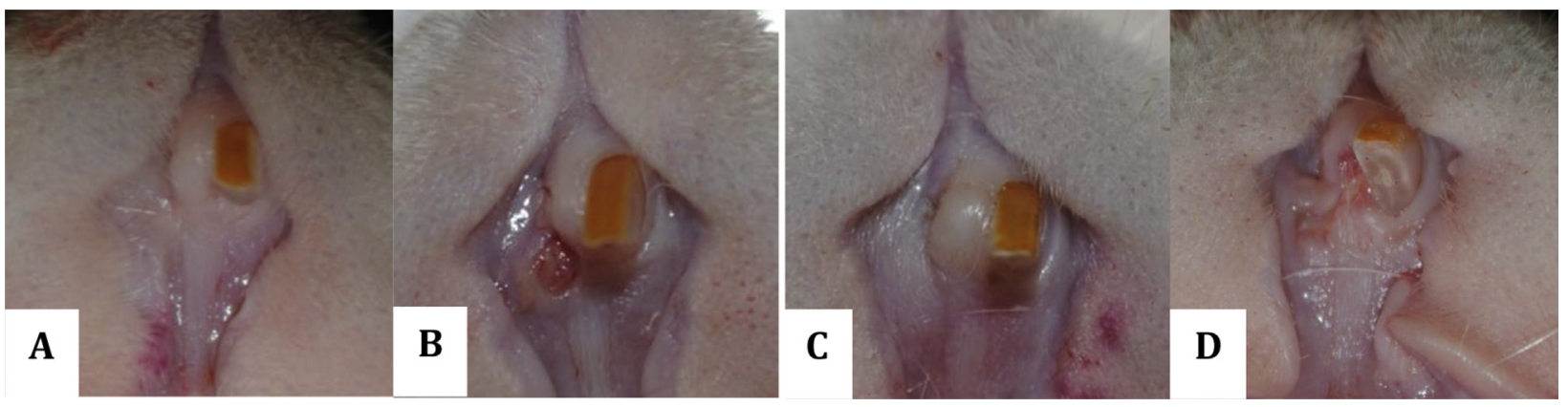

Fig. 1. Clinical aspect of the healing sockets at day 14 of Control (A) and ZL (B), and at day 28, Control (C) and ZL (D) groups.

region, intense focal infiltration by neutrophils (polymorphous) was noted. Surrounding the trabecullae there was highly vascularized loose connective tissue also presenting mononuclear leukocytes. Blood clots were seen distributed all over the sockets. At day 28, mature bone trabecullae were seen among immature ones, associated to osteoclastic activity. Osteoclasts could be seen in the entire socket, usually located inside Howship lacunas; however, most of them were distant from bone surface, presenting a round shape and not associated to the BMUs. Highly vascularized loose connective tissue was surrounding bone trabecullae, along with focal blood clots distributed in the entire socket (Fig. 3).

\subsection{Immunohistochemistry}

Runx-2 - Intense immunolabeling was observed in osteoblasts and eventual cells of granulation tissue considering the entire socket of the $\mathrm{C}$ group. ZL showed moderate labeling also in the osteoblasts, especially in the medium and apical regions, where new bone formation was more evident. At day 28, the $\mathrm{C}$ group presented moderate immunolabeling, detected in osteoblasts associated to remodeling trabecullae, whereas ZL showed mild to moderate labeling in osteoblasts and connective tissue cells.

OC - Moderate labeling of the $C$ group at day 14 was observed predominantly in the areas of mature bone matrix, and also in osteoblast cytoplasm related to active orthogenesis, while the $\mathrm{ZL}$ group presented mild immunolabeling. At day 28, moderate immunolabeling was maintained in the $C$ group, more evident in mature trabecullae, osteoblasts, and osteocytes, whereas ZL presented moderately, especially in apical and medium regions, eventually in osteoblasts and osteocytes.

$O P G$ - Intense labeling was noted in the $C$ group at day 14 , predominantly in the osteocytes of newly formed trabecullae. In the ZL group, it was moderate, predominantly in apical and medium regions in osteocytes and granulation tissue cells. At day 28, intense immunolabeling was maintained in the $C$ group, in connective cells, osteoblasts, and osteocytes. Meanwhile, the ZL group showed mild labeling, eventually in osteocytes.

$R A N K L$ - At days 14 and 28, intense labeling was observed in the $C$ group, predominantly in osteocytes and osteoblasts, as well as in the ZL group.

Immunolabeling is presented in Fig. 4. Predominant scores of each protein are presented in Table 1.

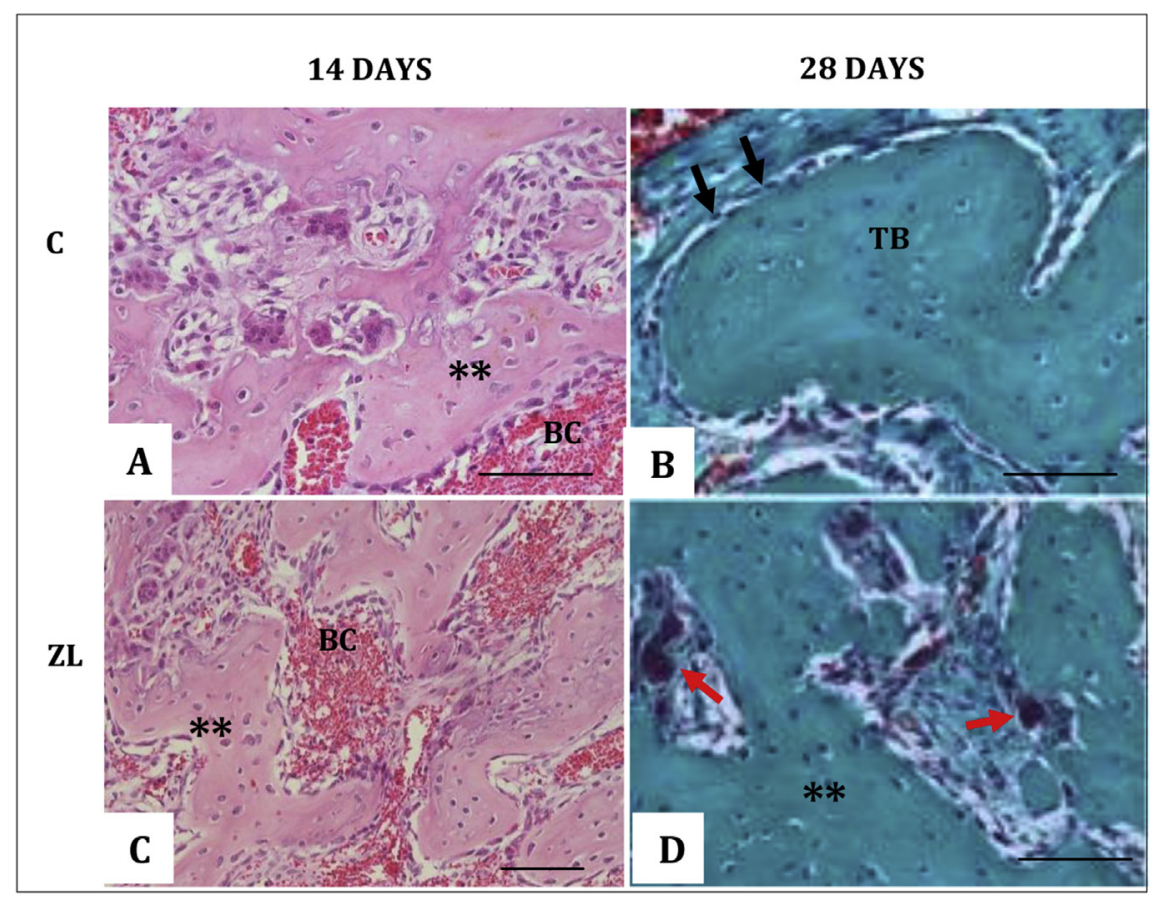

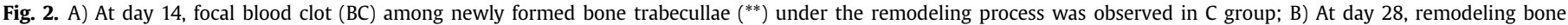

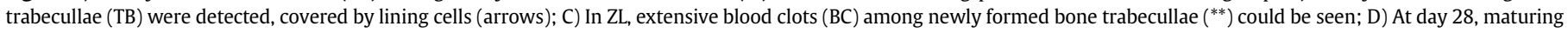
bone trabecullae $\left(^{* *}\right)$ and intense osteoclastic activity (red arrows) were detected $(\mathrm{A}, \mathrm{C}=\mathrm{HE} ; \mathrm{B}, \mathrm{D}=\mathrm{Goldner}$ Trichrome; bar $=150 \mu \mathrm{m})$. 


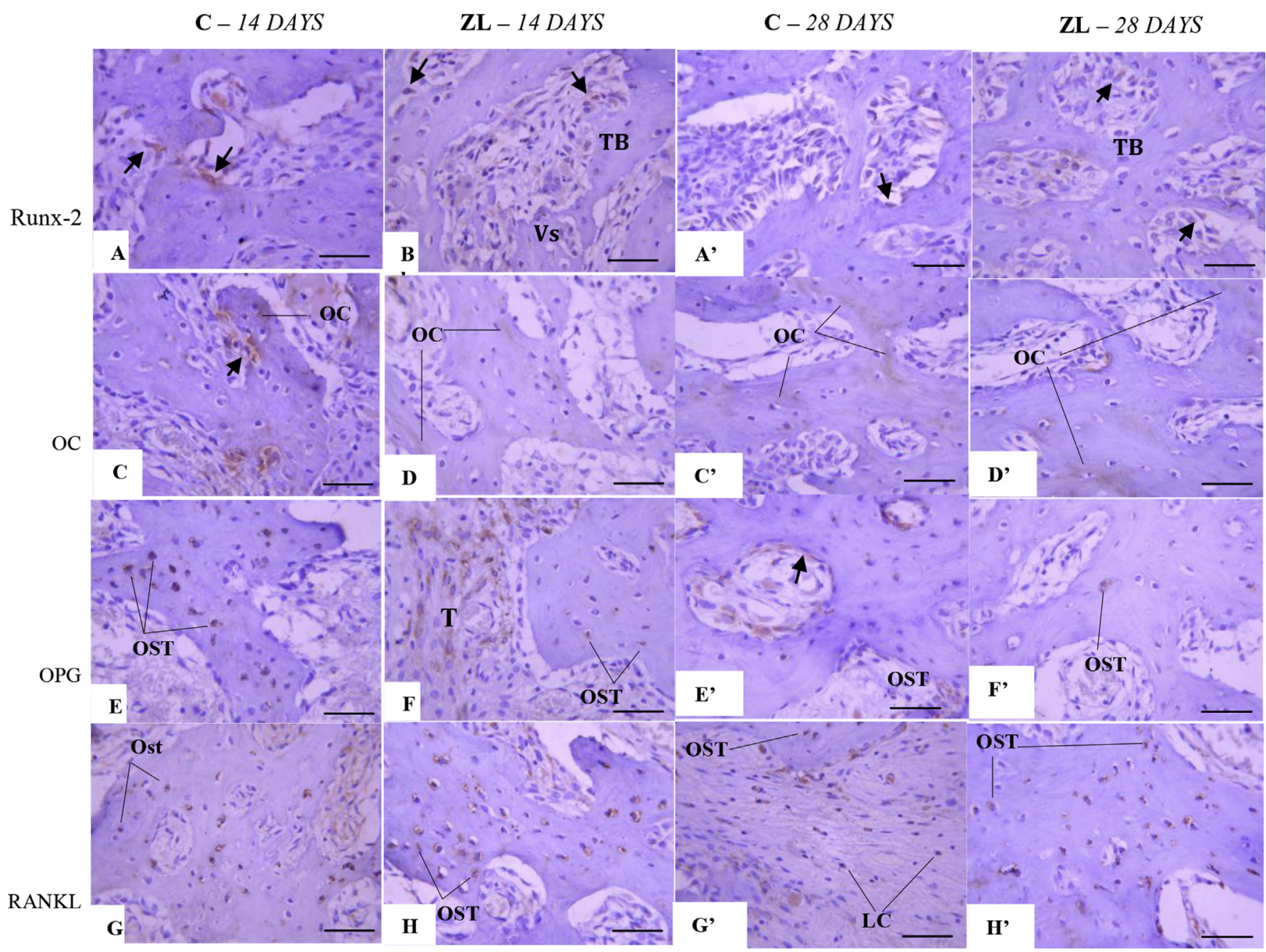

Fig. 3. (A-H) 14 days - Intense Runx-2 osteoblasts immunolabeling (arrows) in C and ZL groups. OC labeling in bone matrix (OC) and osteoblasts (arrows). Marked osteocytes (OST) with OPG and RANKL labeling. ( $\left.\mathrm{A}^{\prime}-\mathrm{H}^{\prime}\right)-28$ days - Moderate Runx-2 labeling in osteoblasts (arrows). Intense OPG immunolabeling in osteocytes (OST) and osteoblasts (arrows) of $\mathrm{C}$ group, while mild in ZL group. Intense RANKL osteocytes (OST) and leukocytes (LC) labeling (bar $=100 \mu \mathrm{m}$ ).

C

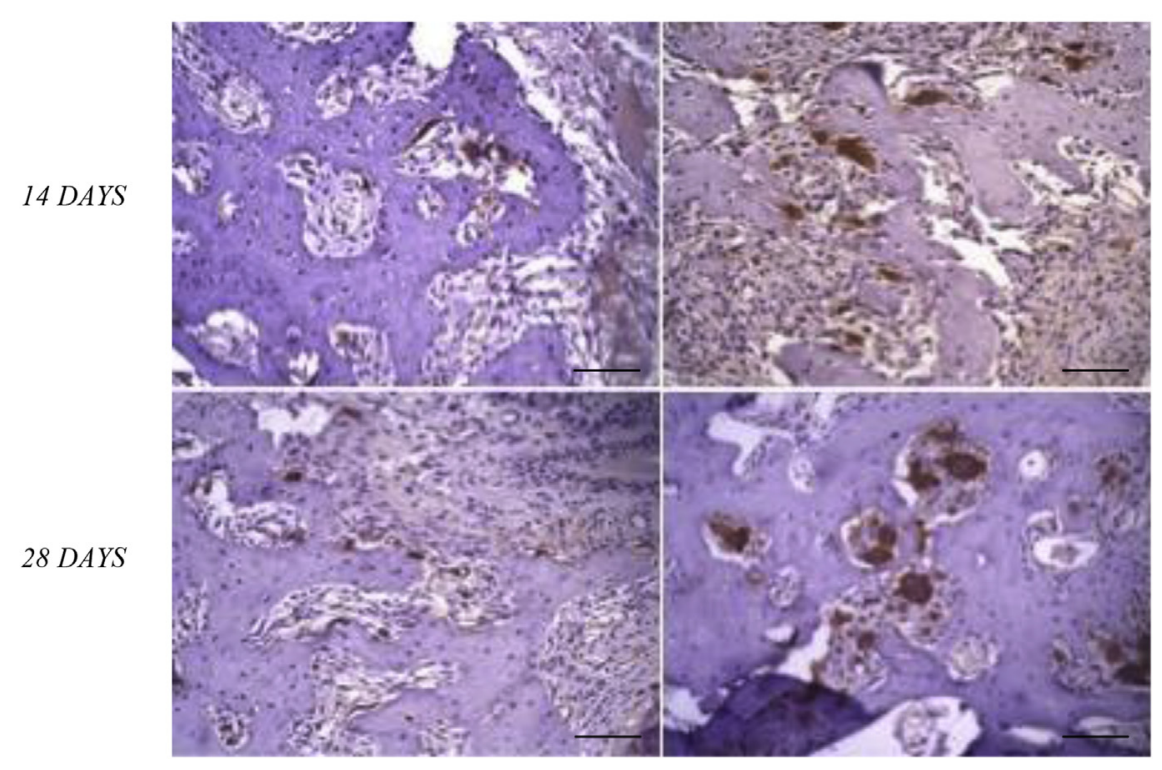

Fig. 4. TRAP - Elongated and irregular TRAP+ cells were observed, located on bone surface in C group at days 14 and 28. ZL group presented round and large cells, most of them unattached to the bone surface, especially at day $28(\mathrm{bar}=100 \mu \mathrm{m})$. 
Table 1

Mean scores assigned for immunohistochemistry markers.

\begin{tabular}{cllll}
\hline & RUNX-2 & OC & OPG & RANKL \\
\hline $\mathbf{1 4}$ days & & & & \\
Control & +++ & ++ & +++ & +++ \\
ZL & ++ & + & ++ & +++ \\
$\mathbf{2 8}$ days & & & & +++ \\
Control & ++ & ++ & +++ & +++ \\
ZL & + & ++ & + & ++ \\
\hline
\end{tabular}

\subsection{Morphometric analysis}

\subsubsection{TRAP + cells (osteoclasts)}

In a general analysis, osteoclasts presenting distinct morphologies were observed comparing the groups. In the $C$ group, they were predominantly thin and elongated, adhered to bone surface in the Howship lacunae. In the ZL group, it was noted that some of them were similar to the $C$ group, but most were large and rounded, and not adhered to bone surface (Fig. 5). Considering this situation, osteoclasts were quantified in their totality (total osteoclasts) and distinctly, adhered and non-adhered. In the comparison of the groups, ZL presented a significant increase in comparison to the $\mathrm{C}$ group ( $p<0.001$ ). Considering the periods, a significantly higher mean number of osteoclasts was detected at day $28(\mathrm{p}=0.049)$, whereas specifically in the $C$ group, no significant differences were identified between the periods ( $p=0.789$ ). However, significant differences were identified in the ZL group when comparing the

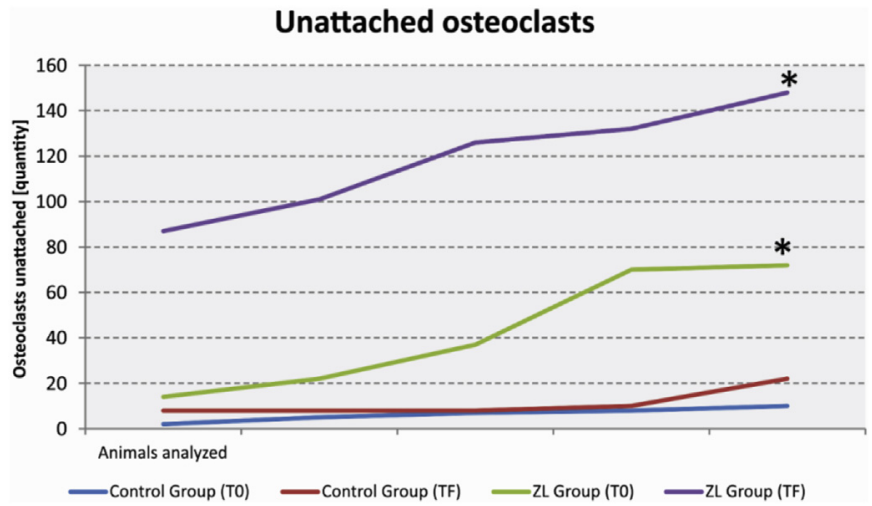

Fig. 5. Statistical analysis for unattached osteoclasts (TRAP+ cells).

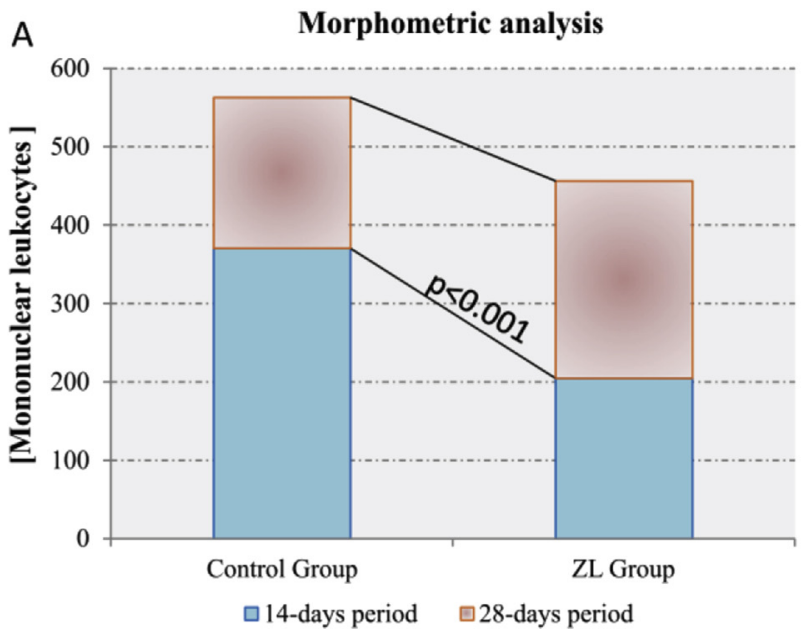

periods $(\mathrm{p}=0.015)$. Analyzing non-adhered osteoclasts, a significant difference was detected between the groups $(p<0.001)$ and periods $(\mathrm{p}<0.001)$. Tukey post-test indicated a significant difference when comparing the groups $(p<0.05)$, with a mean number of 8.8 for the $C$ group, and 80.9 for ZL. Specific analysis inside the $C$ group for the different periods did not detect a significant difference between days 14 and $28(\mathrm{p}=0.687$ ). However, in the specific analysis of the ZL group, a significant increase at day 28 was identified ( $p<0.001$ ), as shown in Fig. 5.

\subsection{Mononuclear leukocytes}

In the comparison of the two groups from the analysis of mononuclear leukocytes quantification, a significant statistical difference was detected only at the 14-days period ( $p<0.001$ ). In the comparison of the periods 14 and 28 days, a significant difference was observed $(p=0.036)$. The control group presented the highest mean of mononuclear leukocytes (281.5) in comparison to ZL (228.1), being statistically significant $(p<0.05)$ in the comparison of the day 14 (mean - C: 370.6 and ZL: 204.4). Specific analysis of the $C$ group detected a significant difference comparing the periods $(\mathrm{p}<0.001)$. In the analysis of $\mathrm{ZL}$, no significant differences were detected in the comparison of the periods $(\mathrm{p}=0.257)$, observing a mean increase of the leukocytes at day 28 (mean 251.8) in comparison to day 14 (mean 204.4), as shown in Fig. 6A.

\subsection{Polymorphonuclear leukocytes}

In the comparison of the groups, a significant difference was detected only at day $14(\mathrm{p}=0.007)$, with an increase in the $C$ group (Control: 173.8; ZL group: 104.0). No significant differences were observed at day $28(\mathrm{p}=0.162)$. In a specific analysis of the $\mathrm{C}$ group, significant reduction $(\mathrm{p}<0.001)$ was observed at day $14(173.8)$ when compared to day 28 (60.6). In the analysis of the ZL group, no differences were detected when comparing the periods $(\mathrm{p}=0.650)$, as shown in Fig. 6B.

\section{Discussion}

Although the results revealed a delayed process on socket healing of aged rats, intravenous therapy of zoledronic acid did not significantly impair it in order to evolve to ONJ.

Among the various experimental studies about the effects of $n-$ BPs on the oral cavity, the preference for rodents is evident (Barba-

B

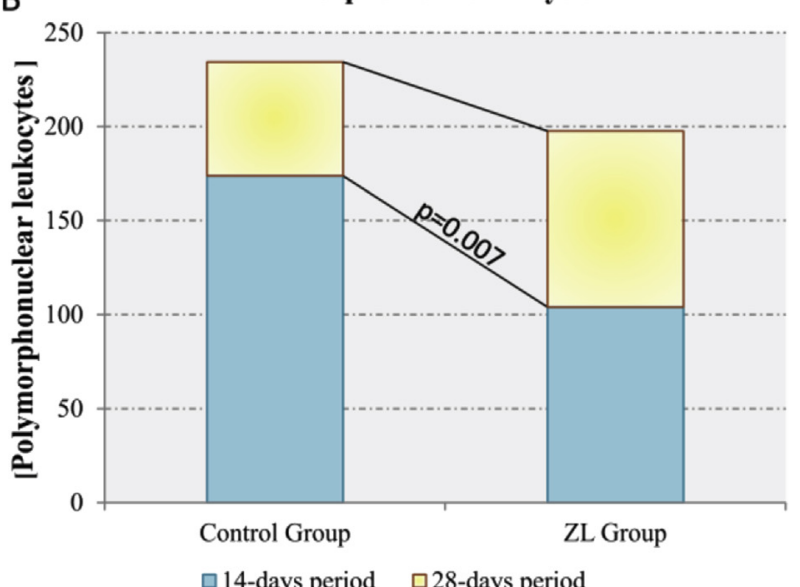

Fig. 6. Statistical results for mononuclear (A) and polymorphonuclear (B) leukocytes considering the analyzed periods and groups. 
Recreo et al., 2014). In the present study, 20-month-old rats were used because musculoskeletal development is finished (Quinn, 2005), corresponding to a human being around 50 years old (Andreollo et al., 2012), while 4 weeks is the mean age in most of the experimental models. Since these are short-lived animals, a few weeks can make difference in terms of metabolism (Sengupta, 2013).

In a comparison with a previous study performed by our research group (Curra et al., 2016), the same methodology was adopted using young rats presenting at three months old, in which ONJ onset was clinically and histologically detected comprising $40 \%$ and $60 \%$, respectively. Intense inflammatory response was installed, with suppuration and bone exposure, as well as bone sequestrum. Since our results could not confirm the diagnosis of ONJ, senescence may have been the differential parameter. Gross evaluation of the $C$ group evidenced no bone exposure and complete healing after 28 days. At days 14 and 28, 4 out of 7, and 2 out of 7 rats of the ZL group presented an open wound, respectively. Despite the fact that soft tissue was not completely healed in these animals, no bone exposure was observed.

Although a lack of significant difference between the C and ZL groups was detected considering the quantity of both polymorphous and mononuclear leukocytes, and the analyzed periods, the $C$ group presented a significant decrease from day 14 to 28 . However, ZL maintained the same mean value of both types of leukocytes, indicating a continued inflammatory process at the late period. This may have influenced the OPG-RANKL ratio imbalance, which presented predominant RANKL immunolabeling in relation to OPG in the ZL group in both periods, becoming more evident at day 28 , when OPG immunolabeling turned mild. Much controversy exists about this question in the literature. While some in vitro studies using hMSC-derived osteoblast cells treated with n-BPs have demonstrated increased expression of OPG and decreased levels of RANKL-inducible genes (Ohe et al., 2012), others have revealed increased expression of RANK and RANKL genes, TNFRSF11A and TNFSF11, respectively, and a decrease in OPG gene, TNFSF11B, by RT and RT-PCR (Nakagawa et al., 2015).

Interestingly, osteoclasts drew attention because of their prevalence and morphology. Morphometric assessment revealed a prevalence of these cells in the ZL group in both periods, contrary to what is expected when using n-BP. However, it is important to emphasize that, aside from the increased number of osteoclasts under ZL action, they presented a distinct morphology and location from those of the group. In the ZL group they were larger, presenting innumerous nuclei, along with a round-shape morphology, and were non-adhered to bone surface. Although some questions still remain about the actual mechanism of action of n-BPs, this evidence is in agreement with the investigations that point out their action on osteoclast cytoskeleton. Rogers et al. (2011) summarized the main studies about the molecular mechanisms of nBPs action on bone resorption, revealing that the internalization of the drug by the osteoclasts strongly inhibits farnesyl pyrophosphate synthase (FPP), responsible for the mevalonate pathway which synthesizes isoprenoid lipids, directly involved in the osteoclast cytoskeletal organization and the formation of ruffled border. Thus, one can conclude that the highest number of these cells in the ZL group is probably explained by their incapacity of organizing their ruffle border, and consequently stabilization on bone surface to resorb bone matrix, making necessary the activation of more pre-osteoclasts and explaining intense RANKL immunolabeling in this group, especially at day 28 .

Although not numerically confirmed, some deficiency might have happened regarding new bone formation by the analysis of Runx-2 labeling, which remained less intense in comparison to the $C$ group. In addition, OC labeling was differently marked when comparing the groups. While it was observed throughout the sockets in the C group, in ZL it was noted mainly in the periphery, confirming a delayed maturation of the newly formed bone. Clinical aspects of the overlying mucosa, which was not completely healed at day 28 in some animals, could also attest delayed repair of ZL extracted sockets.

Relevant information on bone senescence has been reported on a physiological basis (Marie, 2014); nonetheless, little is available when it comes to bone repair (Alleyene et al., 2016; Ishijima et al., 2016). Interestingly, evidence of age-related resistance of rats to parasites has been reported, revealing a better immune system response of adult rats in comparison to young ones (Pascutti et al., 2003). If this is applicable to a condition at high risk of infection, it may be taken as a hint for further investigations in this field.

\section{Conclusions}

From the study results, it can be concluded that IV administration of ZL delayed socket bone repair of aged rats, causing OPGRANKL system imbalance, resulting in deficient bone forming and remodeling, alteration of osteoclasts morphology, as well as maintaining continued inflammation during the healing period. However, ONJ onset could not be attested, since classic clinical and histological aspects were not observed. Nonetheless, the present experimental model raises a question about different responses to IV BP considering animal age.

\section{Funding}

This work was supported by Pró-Reitoria de Pesquisa (PROPe) UNESP, grant number 671.

\section{Acknowledgements}

The authors are grateful to Maira Cristina Rondina Couto from Sagrado Coração University, for histology and imunohistochemistry assistance.

\section{References}

Adam E, Pierrot C, Lafitte S, Godin C, Saoudi A, Capron M, et al: The age-related resistance of rats to Plasmodium berghei infection is associated with differential cellular and humoral immune responses. Int J Parasitol 33: 1067-1078, 2003

Allen MR, Gineyts E, Leeming DJ, Burr DB, Delmas PD: Bisphosphonates alter trabecular bone collagen cross-linking and isomerization in beagle dog vertebra. Osteoporos Int 19: 329-337, 2008

Alleyne B, Varghai D, Askeroglu U, Zwiebel S, Tobin K, Gosain AK: The impact of age upon healing: absolute quantification of osteogenic genes in calvarial criticalsized defects. J Craniofac Surg 27: 258-263, 2016

Andreollo NA, Santos EF, Araújo MR, Lopes LR: Rat's age versus human's age: what is the relationship? Arq Bras Cir Dig 25: 49-51, 2012

Barba-Recreo P, Del Castillo Pardo de Vera JL, García-Arranz M, Yébenes L, Burgueño M: Zoledronic acid-related osteonecrosis of the jaws. Experimental model with dental extractions in rats. J Craniomaxillofac Surg 42: 744-750, 2014

Chan CK, Mason A, Cooper C, Dennison E: Novel advances in the treatment of osteoporosis. Br Med Bull 119: 129-142, 2016

Curra C, Cardoso CL, Ferreira Júnior O, Curi MM, Matsumoto MA, Cavenago BC, et al: Medication-related osteonecrosis of the jaw. Introduction of a new modified experimental model. Acta Cir Bras 31: 308-313, 2016

Hokugo A, Christensen R, Chung EM, Sung EC, Felsenfeld AL, Sayre JW, et al: Increased prevalence of bisphosphonate-related osteonecrosis of the jaw with vitamin D deficiency in rats. J Bone Miner Res 25: 1337-1349, 2010

Ishijima M, Ghassemi A, Soltanzadeh P, Tanaka M, Nakhaei K, Park W, et al: Effect of UV photofunctionalization on osseointegration in aged rats. Implant Dent. 25(6): 744-750, 2016 Dec

Luvizuto ER, ias SM, Queiroz TP, Okamoto T, Garcia Jr IR, Okamoto R, et al: Osteocalcin immunolabeling during the alveolar healing process in ovariectomized rats treated with estrogen or raloxifene. Bone 46: 1021-1029, 2010

Manolagas SC, Parfitt AM: What old means to bone. Trends Endocrinol Metab 21: 369-374, 2010

Marie PJ: Bone cell senescence: mechanisms and perspectives. J Bone Miner Res 29: 1311-1321, 2014 
Marx RE: Pamidronate (Aredia) and zoledronate (Zometa) induced avascular necrosis of the jaws: a growing epidemic. J Oral Maxillofac Surg 61: 1115-1117, 2003

Nakagawa T, Ohta K, Kubozono K, Ishida Y, Naruse T, Takechi M, et al: Zoledronate inhibits receptor activator of nuclear factor kappa-B ligand-induced osteoclast differentiation via suppression of expression of nuclear factor of activated T-cel c1 and carbonic anhydrase 2. Arch Oral Biol 60: 557-565, 2015

Ohe JY, Kwon YD, Lee HW: Bisphosphonates modulate the expression of OPG and M-CSF in hMSC-derived osteoblasts. Clin Oral Investig 16: 1153-1159, 2012

Ostan R, Monti D, Gueresi P, Bussolotto M, Franceschi C, Baggio G: Gender, aging and longevity in humans: an update of an intriguing/neglected scenario paving the way to a gender-specific medicine. Clin Sci (Lond) 130: 1711-1725, 2016

Pascutti MF, Bottasso OA, Hourquescos MC, Wietzerbin J, Revelli S: Age-related increase in resistance to acute Trypanosoma cruzi infection in rats is associated with an appropriate antibody response. Scand J Immunol 58: 173-179, 2003

Pazianas M, Abrahamsen B: Osteoporosis treatment: bisphosphonates reign to continue for a few more years, at least? Ann N Y Acad Sci 1376: 5-13, 2016
Pelaz A, Junquera L, Gallego L, García-Consuegra L, García-Martínez L, Cutilli T, et al: Epidemiology, pharmacology and clinical characterization of bisphosphonaterelated osteonecrosis of the jaw. A retrospective study of 70 cases. Acta Otorrinolaringol Esp 66: 139-147, 2015

Quinn R: Comparing rat's to human's age: how old is my rat in people years? Nutrition 21: 775-777, 2005

Rogers MJ, Crockett JC, Coxon FP, Mönkkönen J: Biochemical and molecular mechanisms of action of bisphosphonates. Bone 49: 34-41, 2011

Ruggiero SL, Dodson TB, Assael LA, Landesberg R, Marx RE, Mehrotra B: Task force on bisphosphonate-related osteonecrosis of the jaws, American Association of Oral and Maxillofacial Surgeons. American Association of Oral and Maxillofacial Surgeons position paper on bisphosphonate-related osteonecrosis of the jaw 2009 update. Aust Endod J 35: 119-130, 2009

Sengupta P: The laboratory rat: relating its age with humans. Int J Prev Med 4: 624-630, 2013

Turner B, Drudge-Coates L, Ali S, Pati J, Nargund V, Ali E, et al: Osteonecrosis of the jaw in patients receiving bone-targeted therapies: an overview-Part I. Urol Nurs 36: 111-116, 2016154 